Tropical Journal of Pharmaceutical Research March 2016; 15 (3): 527-533

ISSN: $1596-5996$ (print); 1596-9827 (electronic)

(C) Pharmacotherapy Group, Faculty of Pharmacy, University of Benin, Benin City, 300001 Nigeria.

All rights reserved.

Available online at http://www.tjpr.org

Original Research Article

http://dx.doi.org/10.4314/tjpr.v15i3.14

\title{
Synergistic Effect of Trehalose and Saccharose Pretreatment on Maintenance of Lyophilized Human Red Blood Cell Quality
}

\author{
Yan-Qiong Li ${ }^{1 \star}$, Rui Hu ${ }^{2}$, Li-Hui Zhong ${ }^{1}$, Qian Sun ${ }^{3}$ and You-Ping Yan ${ }^{1}$ \\ ${ }^{1}$ Department of Transfusion, ${ }^{2}$ Physical Examination Center, ${ }^{3}$ Clinical Laboratory, The Central Hospital of Zhumadian City in \\ Henan Province, Zhumadian 463000, PR China \\ *For correspondence: Email: li_yanqiong63@163.com
}

Received: 1 September 2015

Revised accepted: 3 February 2016

\begin{abstract}
Purpose: To investigate the synergistic effect of trehalose and saccharose pretreatment on maintenance of lyophilized human red blood cell $(R B C)$ quality.

Methods: RBCs were pre-treated with trehalose and saccharose, and then lyophilized and re-hydrated. Prior to lyophilization and after re-hydration, RBC parameters, RBC counts, total hemoglobin concentration, mean corpuscular hemoglobin (MCH), mean corpuscular volume (MCV), comprehensive deformation index, hemolysis ratio and phosphatidylserine (PS) expression, were determined using a hematology analyzer, an RBC deformation instrument, a spectrophotometer and a flow cytometer, respectively. Superoxide dismutase (SOD), glucose-6-phosphate dehydrogenase (G-6-PD), and adenosine triphosphatase (ATPase) activities were determined using kits for SOD, ATPase, and G-6$P D$ assay, respectively.

Results: After lyophilization-rehydration, RBC counts and total hemoglobin recovery rates, deformability, and RBC SOD, ATPase, and G-6-PD activities were significantly decreased by $47.24-$ $74.65 \%(p<0.01)$, compared with the normal group. RBC osmotic fragility and PS expression on the outer surface of the RBC membrane were significantly increased by 168.53 and $629.30 \%(p<0.01)$, respectively, compared with the normal group. RBC MCH and MCV values were not significantly affected by lyophilization-rehydration $(p>0.05)$. Trehalose and saccharose pretreatment significantly reversed the effects of lyophilization-rehydration on these RBC parameters by approximately 13.16 $211.11 \%(p<0.01)$, compared with the control group. The combined effects were synergistic.

Conclusion: Trehalose and saccharose pretreatment synergistically enhances maintenance of lyophilized RBC quality.
\end{abstract}

Keywords: Trehalose, Saccharose, Lyophilization, Red blood cell, Hematological parameters

Tropical Journal of Pharmaceutical Research is indexed by Science Citation Index (SciSearch), Scopus, International Pharmaceutical Abstract, Chemical Abstracts, Embase, Index Copernicus, EBSCO, African Index Medicus, JournalSeek, Journal Citation Reports/Science Edition, Directory of Open Access Journals (DOAJ), African Journal Online, Bioline International, Open-J-Gate and Pharmacy Abstracts

\section{INTRODUCTION}

Human red blood cell (RBC) preservation methods include short-term preservation at $4{ }^{\circ} \mathrm{C}$ for $35-42$ days and long-term preservation at 80 or $-196{ }^{\circ} \mathrm{C}$ for approximately 10 years. Because the two preservation methods require specialized preservation devices and are expensive to perform [1], they do not satisfy clinical and emergency use requirements for RBC. Lyophilization is a new RBC preservation method. Compared with the short- and long-term methods, lyophilization advantages include longterm room temperature preservation, convenient transportation, and simple storage conditions $[2,3]$. However, despite these advantages, 
problems such as membrane damage, hemoglobin leakage, and high RBC hemolysis ratio have been reported [4]. In recent decades, lyophilization protectants have been developed to resolve these problems.

Trehalose and saccharose are two intracellular protectants that can reduce the intracellular water content and increase the concentration of cytoplasm components [1]. When cells are freeze-dried, the hydroxyl functional groups of trehalose and saccharose can combine with cell proteins and lipids via hydrogen bonds; this change maintains the stability of cell membranes and proteins [5]. Trehalose and saccharose have been used as lyophilization protectants in many fields [6-8], but the synergistic effects of trehalose and saccharose pretreatment on the maintenance of lyophilized RBC quality are unknown. This study was designed to investigate whether trehalose and saccharose pretreatment can synergistically reverse the effects of lyophilization-rehydration on the maintenance of lyophilized RBC quality.

\section{EXPERIMENTAL}

\section{Chemicals and reagents}

Acid citrate-dextrose (ACD) anticoagulant and PBS buffer solution were obtained from SigmaAldrich (St. Louis, MO, USA). The lyophilization buffer solution consisted of $15 \%$ polyvinylpyrrolidone, $15 \%$ bovine serum albumin, $10 \%$ sodium citrate and $20 \%$ trehalose. The re-hydration buffer solution consisted of $111 \mathrm{mmol} / \mathrm{L}$ glucose, $41 \mathrm{mmol} / \mathrm{L}$ mannitol, $2 \mathrm{mmol} / \mathrm{L}$ adenine, and $\mathrm{NaCl}(1.0,0.5$, or $0.14 \mathrm{~mol} / \mathrm{L})$. Annexin V-FITC, superoxide dismutase (SOD), glucose-6-phosphate dehydrogenase (G-6-PD) and adenosine triphosphatase (ATPase) assay kits were obtained from Beyotime Biotechnology (Shanghai, China).

\section{Preparation of RBCs and grouping}

Whole blood that was derived from venous blood of healthy volunteers was obtained from the Department of Transfusion of the Central Hospital of Zhumadian City (Henan Province, China). After pretreatment with ACD anticoagulant, whole blood was centrifuged at $3000 \mathrm{rpm}$ for $10 \mathrm{~min}$ at $4{ }^{\circ} \mathrm{C}$ and the supernatant and buffy coat were discarded to obtain the concentrated RBCs. RBCs were then washed thrice with isotonic PBS and centrifuged at 3000 rpm for 10 min at $4{ }^{\circ} \mathrm{C}$ to obtain standby RBCs. The standby RBCs were suspended in isotonic
PBS buffer solution to provide the $45 \%$ hematocrit RBCs. These RBCs were stored at a temperature of $4{ }^{\circ} \mathrm{C}$ until they were used for the experiment.

The $45 \%$ hematocrit RBCs were randomly divided into 5 groups of $20 \mathrm{~mL}$ each $(\mathrm{n}=10$ replicates per group). The groups were the normal RBC group (NG, fresh RBCs, no lyophilization-rehydration), the control group (CG, no pretreatment with lyophilization-rehydration), the trehalose group (TG, $800 \mathrm{mmol} / \mathrm{L}$ trehalose pretreatment with lyophilization-rehydration), the saccharose group (SG, $800 \mathrm{mmol} / \mathrm{L}$ saccharose pretreatment with lyophilization-rehydration), and the trehalose and saccharose group (TSG, 400 $\mathrm{mmol} / \mathrm{L}$ trehalose and $400 \mathrm{mmol} / \mathrm{L}$ saccharose pretreatment with lyophilization-rehydration).

\section{Trehalose and saccharose pretreatment}

The $20 \mathrm{~mL} 45 \%$ hematocrit RBCs samples were separately pre-treated with different concentrations of trehalose and saccharose as described above. For example, for one pretreatment ( $800 \mathrm{mmol} / \mathrm{L}$ trehalose), $25 \mathrm{~mL} 1.6$ $\mathrm{mol} / \mathrm{L}$ trehalose and $15 \mathrm{~mL}$ isotonic PBS were slowly added to $20 \mathrm{~mL} 45 \%$ hematocrit RBCs. After a 5-min equilibration period, an additional $25 \mathrm{~mL} 1.6 \mathrm{~mol} / \mathrm{L}$ trehalose and $15 \mathrm{~mL}$ isotonic PBS were slowly added to the sample. The final samples were incubated at $37^{\circ} \mathrm{C}$ for $7 \mathrm{~h}$. The RBCs were then washed thrice with isotonic PBS and isolated using centrifugation at $3000 \mathrm{rpm}$ for $10 \mathrm{~min}$ at $4^{\circ} \mathrm{C}$.

\section{Pre-freezing and lyophilization}

Each sample of pre-treated RBCs $(0.3 \mathrm{~mL})$ was re-suspended in $0.7 \mathrm{~mL}$ lyophilization buffer and then transferred into a freeze dry vial and equilibrated for $30 \mathrm{~min}$ at $4{ }^{\circ} \mathrm{C}$. The sample was then cooled to $-70{ }^{\circ} \mathrm{C}$ at a cooling rate of -5 ${ }^{\circ} \mathrm{C} / \mathrm{min}$ in a programmable freezer and was held for $2 \mathrm{~h}$. The vial was then placed on the shelf of a vacuum freeze-drying apparatus (Advantage EL, Virtis, USA) at 150 mbar vacuum pressure. The lyophilization process included two steps (i.e., primary, and secondary drying), which is presented in Table 1.

\section{Re-hydration}

Re-hydration was performed by directly adding pre-heated re-hydration buffer $\left(37^{\circ} \mathrm{C}\right)$ to each lyophilized RBC sample. The sample was completely dissolved using gentle shaking. To decrease osmotic damage of lyophilized RBCs during the lyophilization to re-hydration process, the lyophilized RBCs were washed with gradient 
saline solutions. Each solution contained the rehydration buffer and $1.0,0.5$, or $0.14 \mathrm{~mol} / \mathrm{L} \mathrm{NaCl}$. The RBCs were then isolated using centrifugation at $3000 \mathrm{rpm}$ for $10 \mathrm{~min}$ at $4{ }^{\circ} \mathrm{C}$. The final volume of each sample was $1 \mathrm{~mL}$.

Table 1: Lyophilization process of pre-treated RBCs

\begin{tabular}{lcc}
\hline $\begin{array}{l}\text { Drying } \\
\text { process }\end{array}$ & $\begin{array}{c}\text { Shelf temp } \\
\left({ }^{\circ} \mathbf{C}\right)\end{array}$ & $\begin{array}{c}\text { Hold time } \\
(\text { h) }\end{array}$ \\
\hline Primary & -70 & 1 \\
& -60 & 2 \\
& -50 & 2 \\
& -45 & 2 \\
& -40 & 2 \\
Secondary & -35 & 2 \\
& -30 & 2 \\
& -20 & 2 \\
& -10 & 1 \\
& 0 & 1 \\
\hline
\end{tabular}

\section{Conventional RBC indicators}

Conventional RBC indicators, including RBC counts, total hemoglobin concentration, mean corpuscular hemoglobin (MCH), and mean corpuscular volume (MCV)] were determined using a hematology analyzer (Sysmex XE-2100, Tokyo, Japan). The RBC counts and total hemoglobin recovery rates were calculated as in Eqs 1 and 2.

RBC counts recovery rate $(\%)=\left(N_{1} / N_{2}\right) 100$

$\mathrm{N}_{2}$ and $\mathrm{N}_{1}$ represented RBC counts before lyophilization and after re-hydration, respectively.

Total hemoglobin recovery rate $(\%)=\left(\mathrm{Hb}_{1} / \mathrm{Hb}_{2}\right) 100$ ..... (2)

$\mathrm{Hb}_{2}$ and $\mathrm{Hb}_{1}$ were total hemoglobin concentrations before lyophilization and after rehydration, respectively.

\section{Evaluation of RBC deformability}

RBC deformation capability was determined using an RBC deformation instrument (LBY-BX, China) according to a reported method [9]. An RBC deformation index was determined and a comprehensive RBC deformation index was calculated for each sample.

\section{Osmotic fragility assay}

The RBC osmotic fragility assay was performed according to a reported method [10]. After rehydration, RBC subsamples $(20 \mu \mathrm{L})$ were separately added to varying concentrations of a $4.5 \mathrm{~mL} \mathrm{NaCl}$ solution $(2.5,3,3.5,4,4.5,5,5.5,6$, 6.5 , or $7 \mathrm{~g} / \mathrm{L}$ ). After $30 \mathrm{~min}$, each sample was centrifuged at $2000 \mathrm{rpm}$ for $20 \mathrm{~min}$. The absorbance (A) of the supernatant was measured at a $540 \mathrm{~nm}$ wavelength using a Shimadzu spectrophotometer (UV-9100, Kyoto, Japan). Before lyophilization, each $20 \mu \mathrm{L}$ RBC sample was added to $0.9 \% 4.5 \mathrm{~mL} \mathrm{NaCl}$ solution; the absorbance $\left(A_{0}\right)$ value for the supernatant was used as the value for the blank control. Before lyophilization, each $20 \mu \mathrm{L}$ RBC was added to distilled water and the absorbance $\left(A_{100}\right)$ value of the supernatant was used as the hemolysis control value. The RBC hemolysis ratio was calculated as in Eq 3 .

\section{Hemolysis ratio $(\%)=\left\{(A-A 0) /\left(A_{100}-A_{0}\right)\right\} 100 \ldots$ \\ Determination of membrane phosphatidyl- serine (PS) expression}

After re-hydration, the RBC sample was resuspended in PBS to obtaine a $1 \times 10^{6} / \mathrm{mL}$ suspension. Each $100 \mu \mathrm{L}$ cell suspension was stained with Annexin V-FITC according to the manufacturer's instructions. The stained RBCs were analyzed using a FACSCalibur flow cytometer (BD Bioscience, Franklin Lakes, NJ, USA).

\section{SOD, ATPase, and G-6-PD assays}

SOD, ATPase, and G-6-PD activities were determined using corresponding kits according to the manufacturer's instructions. The SOD activity $(\mathrm{U} / \mathrm{gHb})$ was determined using a xanthine oxidase method [11]. The ATPase activity $\left(\mathrm{U} / 10^{7}\right.$ RBCs) was determined based on ATP-ATPase reaction that generates inorganic phosphate [12]. The G-6-PD activity $(\mathrm{U} / \mathrm{gHb})$ was determined based on NADP ${ }^{+}$-G-6-PD reaction that generates NADPH [13].

\section{Statistical analysis}

All results are presented as mean \pm standard error of the mean (SEM, $n=10$ ). Between-group differences were analyzed using one-way ANOVA (LSD test) on SPSS 21.0 software, IBM Corporation, Armonk, NY, USA. Differences were considered statistically significant at $p<0.05$ or 0.01 .

\section{RESULTS}

\section{Effects of trehalose and saccharose pretreatment on conventional lyophilized RBC indicators}

After lyophilization-rehydration, the RBC counts and total hemoglobin recovery rates in the TG, $S G$, and TSG were significantly increased by $21.24-180.57 \%(p<0.01)$, compared with the 
CG (Fig. 1A). RBC and total hemoglobin recoveries among the TG, SG, and TSG were also significantly different (TSG > SG > TG; $p<$ 0.01 ). The differences in $M C H$ and $M C V$ values among the NG, CG, TG, SG, and TSG were not statistically significant (Fig. 1B).

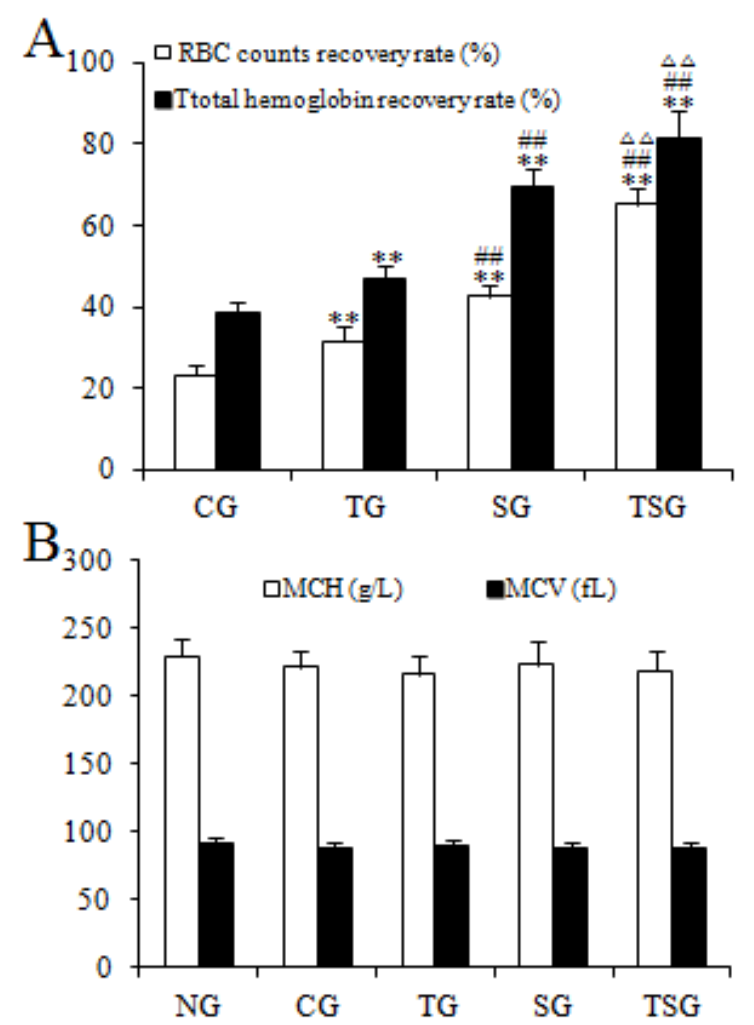

Figure 1: Effects of trehalose and saccharose pretreatment on conventional lyophilized RBC indicators including RBC counts and total hemoglobin recovery rates, $\mathrm{MCH}$ and $\mathrm{MCV} ;{ }^{* *} p<0.01$, compared with the CG; ${ }^{\# \#} p<0.01$, compared with the TG; $\Delta \Delta p<$ 0.01 , compared with the SG

\section{Effects of trehalose and saccharose pretreatment on comprehensive lyophilized RBC deformation index}

The lyophilized RBC comprehensive deformation index in the CG was significantly decreased by $56.25 \%(p<0.01)$, compared with the NG (Fig. 2). After trehalose and saccharose pretreatment, the comprehensive lyophilized RBC deformation indices in the TG, SG, and TSG were significantly increased by $38.10,71.43$ and $100.00 \%(p<0.01)$, respectively, compared with the CG. The comprehensive lyophilized RBC deformation indices among the TG, SG, and TSG were also significantly different (TSG > SG $>$ TG; $p<0.01$ ).

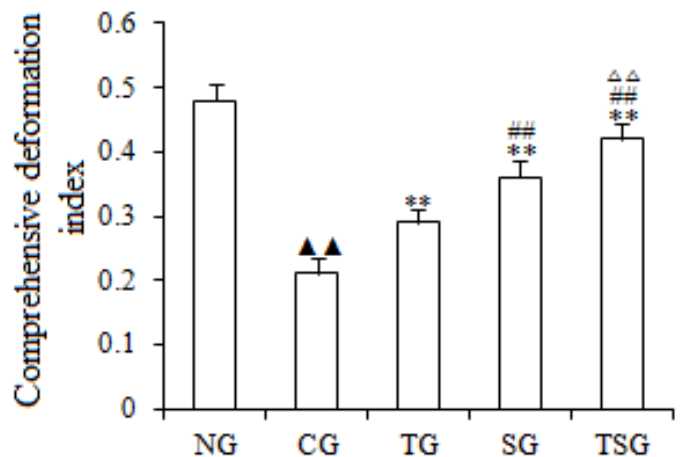

Figure 2: Effects of trehalose and saccharose pretreatment on comprehensive lyophilized RBC deformation index; ${ }^{\Delta \wedge} p<0.01$, compared with the NG; ${ }^{* *} p<0.01$, compared with the CG; ${ }^{\# \#} p<0.01$, compared with the TG; $\Delta \Delta p<0.01$, compared with the SG

\section{Effects of trehalose and saccharose pretreatment on lyophilized RBC hemolysis ratio}

The hemolysis ratio versus $\mathrm{NaCl}$ curve in the $\mathrm{CG}$ was shifted to the right, compared with the NG (Fig. 3A), indicating that the hemolysis ratios were increased in the CG. After trehalose and saccharose pretreatment, the hemolysis ratio versus $\mathrm{NaCl}$ curves in the TG, SG, and TSG were shifted to the left, compared with the CG, suggesting that the hemolysis ratios were decreased in these groups. The statistical analysis of the hemolysis ratios at $4.5 \mathrm{~g} / \mathrm{L} \mathrm{NaCl}$ indicated that the lyophilized RBC hemolysis ratio in the $C G$ was significantly increased by $168.53 \%(p<0.01)$, compared with the NG (Fig. 3B). After trehalose and saccharose pretreatment, the lyophilized RBC hemolysis ratios in the TG, SG, and TSG were significantly decreased by 13.16, 26.81 and $54.57 \%(p<$ 0.01 ), respectively, compared with the $C G$. The lyophilized RBC hemolysis ratios among the TG, SG, and TSG were also significantly different $(\mathrm{TSG}<\mathrm{SG}<\mathrm{TG} ; p<0.01$ ).

\section{Effects of trehalose and saccharose pretreatment on PS expression in lyophilized RBC}

PS expression on the outer surface of lyophilized RBC membrane in the CG was significantly increased by $629.30 \%(p<0.01)$, compared with the NG (Fig. 4). After trehalose and saccharose pretreatment, PS expression in the TG, SG, and TSG were significantly decreased by 44.58 , 52.49 and $70.54 \%(p<0.01)$, respectively, compared with the CG. PS expressions among 
the TG, SG, and TSG were also significantly different (TSG $<\mathrm{SG}<\mathrm{TG} ; p<0.01)$.
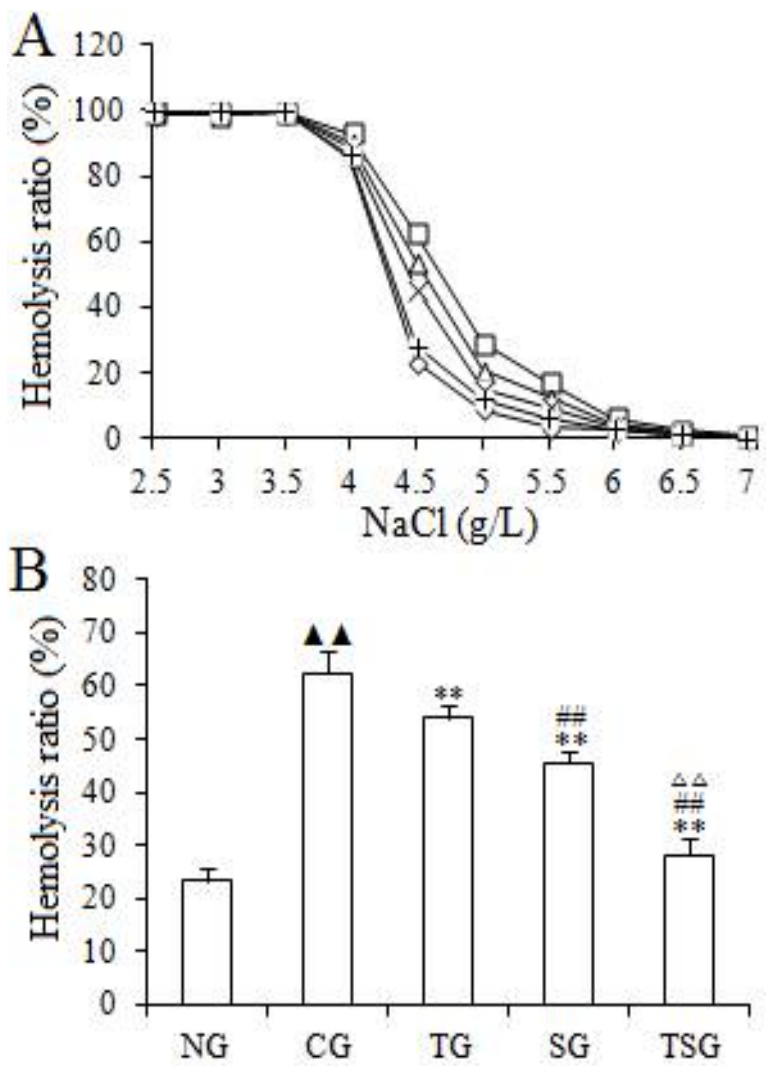

Figure 3: Effects of trehalose and saccharose pretreatment on lyophilized RBC hemolysis ratio; A: Lyophilized RBC hemolysis ratio in the NG $(\diamond), \mathrm{CG}$ $(\square)$, TG $(\Delta)$, SG $(x)$ and TSG $(+)$ when the concentrations of $\mathrm{NaCl}$ were 2.5, 3, 3.5, 4, 4.5, 5, 5.5, 6, 6.5 and $7 \mathrm{~g} / \mathrm{L}$, respectively; B: Lyophilized RBC hemolysis ratio when the concentration of $\mathrm{NaCl}$ was $4.5 \mathrm{~g} / \mathrm{L} ;{ }^{\Delta \Delta} p<0.01$, compared with the NG; ${ }^{* *} p<$ 0.01 , compared with the CG; ${ }^{\# \#} p<0.01$, compared with the TG; $\Delta \Delta p<0.01$, compared with the SG

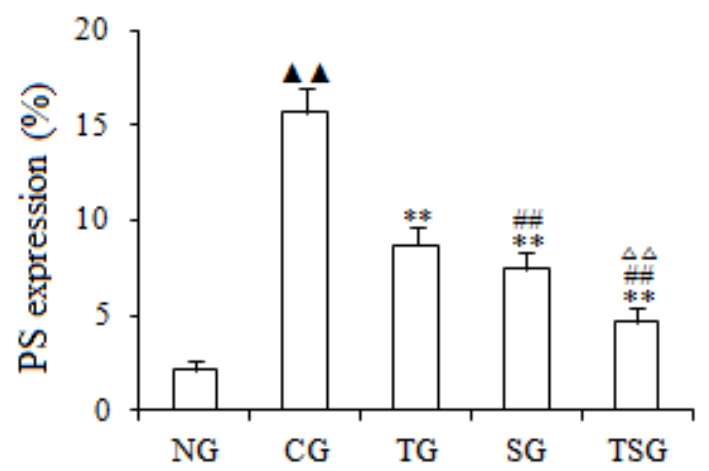

Figure 4: Trehalose and saccharose pretreatment decreased PS expression on the outer surface of lyophilized RBC membrane; ${ }^{\boldsymbol{\Delta} \Delta} p<0.01$, compared with the NG; ${ }^{* *} p<0.01$, compared with the CG; ${ }^{\# \#} p<$ 0.01 , compared with the TG; $\Delta \Delta p<0.01$, compared with the SG
Effects of trehalose and saccharose pretreatment on SOD, ATPase, and G-6-PD activities of lyophilized RBC

The SOD, ATPase, and G-6-PD activities of lyophilized RBC in the CG were significantly decreased by $47.27,49.46$ and $74.65 \%(p<$ 0.01 ), respectively, compared with the NG (Fig. 5). After trehalose and saccharose pretreatment, the SOD, ATPase and G-6-PD activities in the TG, SG, and TSG were significantly increased by $20.21-211.11 \%(p<0.01)$, compared with the CG. The SOD, ATPase, and G-6-PD activities among the TG, SG, and TSG were also significantly different (TSG $>S G>T G ; p<0.01)$.
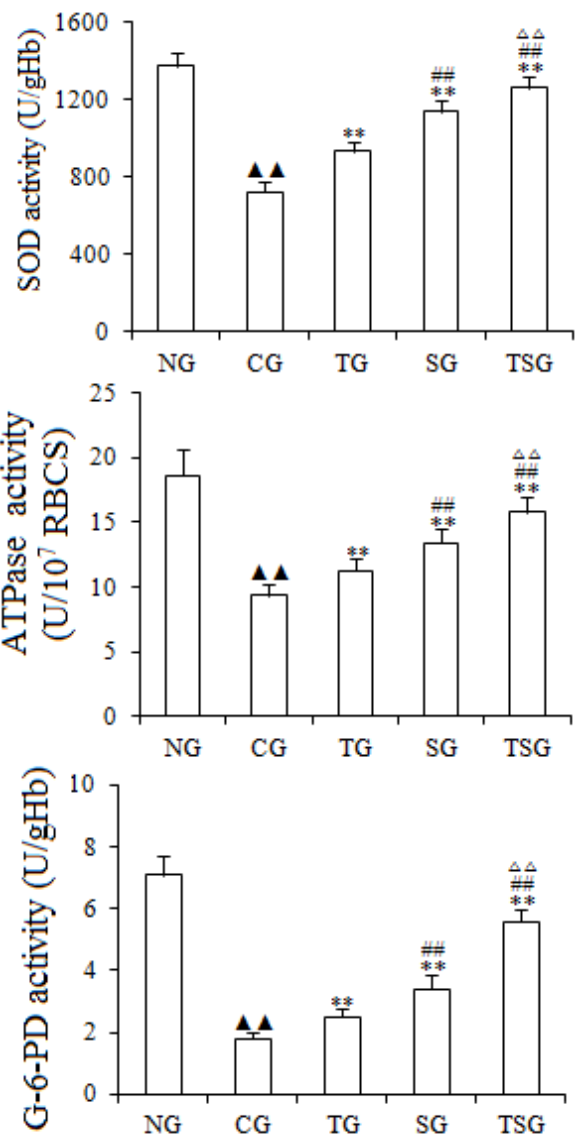

Figure 5: Effects of trehalose and saccharose pretreatment on SOD, ATPase and G-6-PD activities of lyophilized RBC; ${ }^{\Delta} p<0.01$, compared with the $N G ;{ }^{* *} p<0.01$, compared with the CG; ${ }^{\# \#} p<0.01$, compared with the TG; $\Delta \Delta p<0.01$, compared with the SG

\section{DISCUSSION}

Lyophilization has been successfully used to preserve RBC. RBC membrane damage, hemoglobin leakage, high hemolysis ratio, and reduction of ATPase, G-6-PD and SOD activities are common problems that result from RBC 
lyophilization $[4,14]$. The damages result from the water loss that occurs during lyophilization [15]. Fortunately, these problems can be resolved using lyophilization protectants. Trehalose and saccharose are two commonly used lyophilization protectants $[15,16]$, but the synergistic effects of trehalose and saccharose pretreatment on the maintenance of lyophilized RBC quality are unknown. We studied the synergistic effects of trehalose and saccharose pretreatment on the maintenance of lyophilized RBC quality by determining RBC counts recovery rate, total hemoglobin concentration recovery rate, $\mathrm{MCH}, \mathrm{MCV}$, comprehensive deformation index, hemolysis ratio, PS expression, and SOD, ATPase, and G-6-PD activities.

The cell is the basic structure and function unit of human metabolism. Hemoglobin, the main RBC protein, has an important role in carrying oxygen and carbon dioxide [17] and is an NO storage library; hemoglobin is an antioxidant and participates in homeostasis and platelet aggregation [18]. Therefore, the RBC counts and total hemoglobin recovery rates are two acceptable indices used to evaluate the effects of trehalose and saccharose pretreatment on maintenance of lyophilized RBC quality [19]. Trehalose and saccharose pretreatment synergistically decreased lyophilizationrehydration-induced damage on RBC counts and total hemoglobin by increasing the RBC counts and total hemoglobin recovery rates (Fig. 1A). $\mathrm{MCH}$ and $\mathrm{MCV}$ are two conventional indicators used to evaluate RBC function $[20,21]$. $\mathrm{MCH}$ and MCV were not affected by lyophilizationrehydration.

RBC deformability has an important role in the apparent viscosity of blood, microcirculation perfusion, and lifespan of RBCs [22]. Trehalose and saccharose pretreatment synergistically decreased lyophilization-rehydration-induced damage on RBC deformability by increasing the comprehensive deformation index (Fig. 2). Osmotic fragility is an important factor in the maintenance of RBC integrity and normal functions [23]. Trehalose and saccharose pretreatment synergistically decreased lyophilization-rehydration-induced damage on RBC osmotic fragility by reducing the osmotic fragility (Fig. 3). PS is only distributed in the internal side of the lipid double-membrane in the normal cell. When the cell undergoes apoptosis or death, PS is transferred from the inside to the outside of the lipid double-membrane. When that occurs, PS can be evaluated using Annexin VFITC stain [24]. Trehalose and saccharose pretreatment synergistically decreased lyophilization-rehydration-induced damage on RBC membrane by decreasing the PS expression on the outside of the RBC membrane.

Adenosine triphosphate (ATP) is an important product of energy metabolism in RBC. ATP has an important role in the maintenance of the morphology and the RBC rheological characteristics [25]. The pre-condition of the release of ATP energy is that ATP is hydrolyzed by ATPase [26]. SOD is a kind of metal enzyme and is distributed throughout the organism; it has important roles in maintaining the balance between oxidation and anti-oxidation and in scavenging free-radicals of RBC $[22,27]$. RBC G6-PD can catalyze glucose-6-phosphate to generate NAD-PH, which is the coenzyme of glutathione reductase. The reduced glutathione is necessary for the maintenance of hemoglobin stability and RBC membrane integrity [28]. Trehalose and saccharose pretreatment synergistically decreased lyophilizationrehydration-induced damage on the SOD, ATPase and G-6-PD activities of RBCs by increasing their activities (Fig. 5).

\section{CONCLUSION}

Trehalose and saccharose pretreatment synergistically improves the maintenance of lyophilized RBC quality by reversing lyophilization-rehydration-induced damage on RBC parameters. Therefore, trehalose and saccharose may be considered to be a combined lyophilization protectant in the lyophilization of RBC. However, studies on further protective mechanisms of trehalose and saccharose in the lyophilization of RBC need to be investigated in future work.

\section{CONFLICT OF INTEREST}

The authors declare that there is no conflict of interest associated with this work.

\section{CONTRIBUTION OF AUTHORS}

We declare that this work was done by the authors named in this article and all liabilities pertaining to claims relating to the content of this article will be borne by the authors. Yan-Qiong Li conceived and designed the study. Yan-Qiong Li, Rui Hu, Li-Hui Zhong, Qian Sun and You-Ping Yan performed the experiments, collected and analyzed the data. 


\section{REFERENCES}

1. Chen YB, Tian L, Song N, Cheng Q, Liu Z. A review of lyophilized reagent for human red blood cells. Chin $J$ Blood Transfusion 2015; 28(3): 338-341.

2. Yao GH, Luan JF, Ye D, Yan JM, Zhu PY, Lei QH. The effect of red blood cells loaded with combination of trehalose and glucose. J Clin Transfus Lab Med 2010; 12(1): 4-6.

3. Ohtake S, Schebor C, Palecek SP, de Pablo JJ. Phase behavior of freeze-dried phospholipids-cholesterol mixtures stabilized with trehalose. Biochim Biophys Acta 2005; 1713(1): 57-64.

4. Zhou XL, Liu JF, Yuan J, Zhou GY. Loading trehalose by electropermeabilization increased the survival of freezedried red blood cells. Chin J Cell Biol 2009; 31(4): 589 593.

5. Hua ZZ. New technology of freeze-drying. Beijing: Science Press; 2006. 391-96p.

6. Leslie SB, Israeli E, Liqhthart B, Crowe JH, Crowe LM. Trehalose and sucrose protect both membranes and proteins in intact bacteria during drying. Appl Environ Microbiol 1995; 61(10): 3592-3597.

7. Beattie GM, Crowe JH, Lopez AD, Cirulli V, Ricordi C, Hayek A. Trehalose: a cryoprotectant that enhance recovery and preserves function of human pancreatic islets after long term storage. Diabetes 1997; 46(3): 519-523.

8. Zhou XL, He H, Liu BL, Hua TC, Chen Y. Effects of glycerol pretreatment on recovery and antioxidant enzyme activities of lyophilized red blood cells. Cryoletters 2008; 29(4): 285-292.

9. Zhuang Y, Liu JH, Quyang XL, Chen LF, Che J. Effect of $D M S O$ on human red blood cell protection during the period of loading trehalose before lyophilization. Chin J Blood Transfusion 2007; 20(2): 93-96.

10. He H, Liu BL, Hua ZZ, Shen M, Chen Y. Glycerol pretreatment improves the preservation quality of red blood cells by freeze-drying. J Univ Shanghai Sci Technol 2006; 28(5): 413-417.

11. Zhao J, $L i X, L u Z$, Wen J. Factors affecting the determination of superoxide dismutase activity. Food Sci 2010; 31(09): 216-218.

12. Fang ZQ, Wang CF, Wei HR. Effects of mercury and selenium on $\mathrm{Na}+/ \mathrm{K}+-A T P a s e$ activity in Xiphophorus helleri Heckel. Chin J Appl Environ Biol 2006; 12(2): 220-223

13. Jia ZL, Yang FD, Tan GC, Sun W. Preliminary study on analytical method of glucose-6-phosphate dehydrogenase active concentration. Lab Med Clin 2008; 5(2): 65-67.

14. He H, Liu BL, Hua ZZ, Li C, Wu ZZ, Shen M, Chen Y. Experimental study on effect of pretreatment with glycerol upon freeze-drying preservation quality of red blood cells. J Refrig 2006; 27(4): 54-58.
15. Satpathy GR, Torok Z, Bail R, Dwyre DM, Little E, Walker NJ, Tablin F, Crowe JH, Tsvetkova NM. Loading red blood cells with trehalose: a step towards biostabilization. Cryobiol 2004; 49(2): 123-136.

16. Quan GB, Han Y, Liu XZ, Liu A, Jin P, Qi K, Ma EP. Comparison of effect of trehalose and sucrose in lyophilization of human red blood cells. Chin J Blood Transfusion 2003; 16(3): 147-150.

17. Bunn HF, May MH, Kocholaty WF, Shields CE. Hemoglobin function in stored blood. J Clin Invest 1969; 48(2): 311-321.

18. Gladwin MT, Lancaster JR Jr, Freeman BA, Schechter $A N$. Nitric oxide's reactions with hemoglobin: a view through the SNO-storm. Nat Med 2003; 9(5): 496-500.

19. Han Y, Quan GB, Liu XZ, Ma EP, Liu A, Jin P, Cao W. Improved preservation of human red blood cells by lyophilization. Cryobiol 2005; 51(2): 152-164.

20. Radomski MW, Sabiston BH, Isoard P. Development of "sports anemia" in physically fit men after daily sustained submaximal exercise. Aviat Space Envir Med 1980; 51(1): 41-45.

21. Unger KW, Johnson JRD. Red blood cell mean corpuscular volume: a potential indicator of alcohol usage in a working population. Am J Med Sci 1974; 267(5): 281-289.

22. Mohanty JG, Nagababu E, Friedman JS, Rifkind JM. SOD2 deficiency in hematopoietic cells in mice results in reduced red blood cell deformability and increased heme degradation. Exp Hematol 2013; 41(3): 316-321.

23. Soares J, Folmer V, Da Rochha JBT, Nogueira CW. Ebselen exhibits glycation-inhibiting properties and protects against osmotic fragility of human erythrocytes in vitro. Cell Biol Int 2014; 38(5): 625-630.

24. Segawa K, Kurata S, Yanagihashi Y, Brummelkamp TR, Matsuda F, Nagata S. Caspase-mediated cleavage of phospholipid flippase for apoptotic phosphatidylserine exposure. Sci 2014; 344(6188): 1164-1168.

25. Ren SP, Li CY, Liu A, Zheng JH, Zhong SZ, Han Y. Trendency and mathematical model of relationship of blood ATP content with temperature and time of preservation. J Exp Hematol 2005; 13(5): 896-900.

26. Zhou M, Politis A, Davies RB, Liko I, Wu KJ, Stewart AG, Stock $D$, Robinson $C V$. Ion mobility-mass spectrometry of a rotary ATPase reveals ATP-induced reduction in conformational flexibility. Nat Chem 2014; 6: 208-215.

27. Yuan T, Fan WB, Cong Y, Xu HD, Li CJ, Meng J, Bao NR, Zhao JN. Linoleic acid induces red blood cells and hemoglobin damage via oxidative mechanism. Int $J$ Clin Exp Pathol 2015; 8(5): 5044-5052.

28. Francis RO, Jhang J, Hendrickson JE, Zimring JC, Hod $E A$, Spitalnik SL. Frequency of glucose-6-phosphate dehydrogenase-deficient red blood cell units in a metropolitan transfusion service. Transfusion 2013; 53(3): 606-611. 